\title{
NONLINEAR MPC BASED ON MULTI-MODEL FOR DISTILLATION COLUMNS
}

\author{
Bjarne A. Foss ${ }_{\square}^{1}$ Song-Bo Cong \\ Department of Engineering Cybernetics \\ Norwegian University of Science and Technology \\ Trondheim, Norway
}

\begin{abstract}
A multi-model structure for model predictive control has been established for a petroleum distillation column through first principle analysis, and its parameters have been estimated from data generated by a rigorous model. The nonlinear multi-model has been used for state-estimation and prediction in a MPC scheme. The controller has been applied to quality control of a FCCU fractionator. Realistic simulations show that multimodel MPC (MMPC) is an attractive option for nonlinear MPC since the scheme implies a straightforward extension of linear MPC, and the results are promising.

Copyright 1999 IFAC
\end{abstract}

Keywords: Nonlinear Model Predictive Control, Multi-Model, FCCU fractionator

\section{INTRDUCTION}

Model predictive control (MPC) has obtained widespread use in the chemical process industries (Qin and Badgwell, 1996). Even though MPC was originally developed to meet the needs of refining plants, thousands of applications can be found in a wide range of application areas. Complicated and coupled transport mechanisms, strongly coupled inputs and outputs, and a wide operation-range characterize many process plants. This should in principle call for a nonlinear model. MPC is, however, usually based on linear models. Two important reasons for this are:

1. A high-fidelity model is not required in many applications due to the fact that the prime task of the MPC is to always push the process against the constraint(s) that maximize some criterion.

2. The control structure relies on linearizing the process by the use of regulatory control, i.e. MPC acts on the setpoints of the basic control loops instead of the control inputs themselves.

Despite these facts there is a potential for nonlinear MPC as also documented in a recent publication (Qin and Badgewell, 1998).

In this paper we focus on the use of a relatively simple way to introduce nonlinear MPC. Nonlinear MPC is designed by using multi-models (MurraySmith and Johansen, 1997, Johansen and Foss, 1997), which can be viewed as a model-scheduling approach. Further, this approach is investigated through simulation of a rigorous model of a typical refining unit, a FCCU (Fluidized Catalytic Cracking Unit) fractionator (Cong et. al., 1998). This model has been validated against real data collected from an industrial unit.

The contents of this paper are: First, multi-modeling is presented, and a control-relevant multi-model for the FCCU fractionator is developed. Second, the nonlinear MPC is formed utilizing relatively

\footnotetext{
${ }^{1}$ Bjarne.Foss@itk.ntnu.no
} 
standard criteria and non-equality constraints. Third, results are presented for a set of realistic scenarios. The paper ends with some conclusions.

\section{MULTI-MODELS}

\subsection{Basic Concept}

A potentially attractive method for dueling with nonlinear processes over a wide operating range is the use of a multi-models. Multi-models can briefly be described as follows.

Consider a process described by a state-space model in the following form

$$
\begin{aligned}
& x=f(x, u) \\
& y=g(x, u)
\end{aligned}
$$

Let the operating point $\phi \in \Phi$ be defined by

$$
\phi=h(x, u, y), \phi \in \Phi
$$

and define an operation regime by a subset $\Phi_{\mathrm{i}} \subseteq \Phi$, $\Phi_{1} \cup \ldots \cup \Phi_{\mathrm{N}}=\Phi$ and $\Phi_{\mathrm{i}} \cap \Phi_{\mathrm{j}}=\varnothing$. This means that the operating set $\Phi$ is partitioned into disjunct operating regimes. To each operating regime we allocate a local model $\left(M_{\mathrm{i}}\right)$. Hence, we define $N$ local models $M_{1}, \ldots, M_{\mathrm{N}}$. A local model can often be simple since it is only valid in one operation regime (and possibly its neighborhood). In this work we will choose the local models as linear state-space models with a structure based on first principles insight of the process in question.

The local linear models are combined into a global nonlinear model $(M)$ by a convex combination of weighting functions $w_{l}(\phi), \ldots, w_{N}(\phi)$.

$$
\begin{gathered}
M \stackrel{\text { def }}{=} \sum_{i=1}^{N} w_{i}(\phi) M_{i} \\
\sum_{i=1}^{N} w_{i}(\phi) \equiv 1, \forall \phi \in \Phi \\
w_{i}(\phi) \in[0,1]
\end{gathered}
$$

Note that model $M_{i}$ will be dominating model when $\phi \in \Phi_{i}$. This means that $w_{i}(\phi)$ will be large (close to one) in this case. A typical choice for $w_{i}(\phi)$ is a normalized Gaussian function.

The development of a multi-model can be divided into four steps. First, the operating points or scheduling variable $\phi$ must be chosen. Typical choices are one or more control inputs $u$, and/or one or more outputs $y$. Second, the system's operating set is decomposed into $N$ operating regimes that completely cover the relevant range of operation. Third, for each operating regime, a local model structure must be developed. Finally, the weighting functions $w_{1}, w_{2}, \ldots, w_{\mathrm{N}}$ are chosen and the model parameters, typically some of the local model parameters, are estimated on the basis of data.

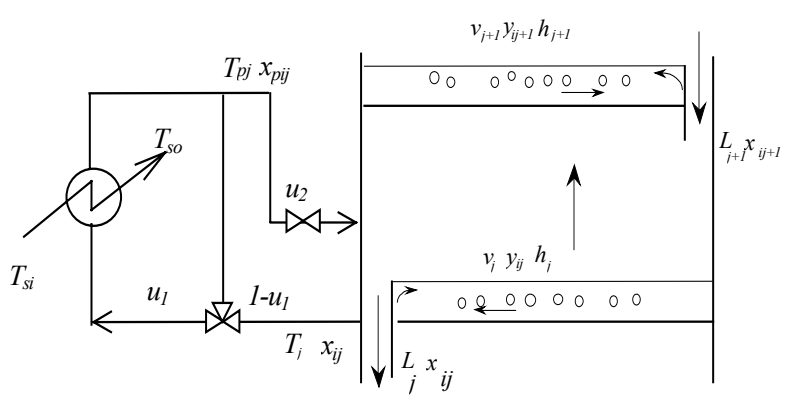

Fig. 1 Tray and pump-around system

\subsection{Model Structure Analysis}

Petroleum distillation column is a well known nonlinear processes. Continuous composition distribution, a large operating range and simultaneously mass and energy transfer contribute to the necessity for a nonlinear high dimension dynamic model.

In a distillation column, even for the single tray shown in Fig. 1, the model dimension can be large, depending on the number of components (Cong et. al., 1998). Obviously, model simplification is needed to obtain a suitable local model for MPC. In order to get a simple structure, we have made the following assumptions:

(1) A number of trays are lumped into one compartment (Benallou, Seborg and Mellichamp, 1986).

(2) The variation of mass holdup $M_{j}$ is neglected, i.e. a constant mole flow-rate is assumed.

(3) Fluid enthalpy is independent of its composition, and is a linear function of temperature.

(4) The heat exchanger in the pump-around section is taken as a lumped system (Cong et. al., 1996). For simplification, the temperature of the medium that exchanges heat with the pumparound fluid is assumed to be constant.

(5) The dynamics of the vapor phase is neglected.

Based on the above assumptions the rigorous dynamic formulations for energy balance in a compartment is as follows:

$$
\begin{aligned}
M_{j}^{L} C_{P}^{L} \frac{d T_{j}}{d t} & =L_{j+1} C_{P}^{L}\left(H_{j+1}-H_{j}\right)+v_{j-1} C_{P}^{V}\left(h_{j-1}-h_{j}\right) \\
& -Q_{j}+L_{P j}\left(u T_{p j}+(1-u) T_{j}-T_{j}\right)
\end{aligned}
$$

If there is a pump-around system connected to the compartment, we have,

$$
M_{h} C_{p}^{L} \frac{d T_{P j}}{d t}=C_{p}^{L}\left(T_{j}-T_{P j}\right) L_{p j}-K_{s t} \Omega\left(T_{p j}-T_{s i}\right)
$$

Thus, for control proposes, the local model of each compartment is simply a first or second order lowpass model. Depending on the pump-around system, petroleum product quality is mainly represented by its cut-point that depends on the operating 
temperature in the tray where it is drawn from. Therefore, the temperature model described above can also be used to control product quality, if this cut-point can be measured or calculated on-line.

Generally, cut points (or temperatures) are controlled within some narrow range in order to meet the product quality requirement, which means that the operating temperature at the tray with production withdraw will not be allowed to change significantly. This operating specification results in a small influence of operation temperature on system nonlinearities.

Feed temperature is commonly controlled by a preheat system or in an up-stream unit. Furthermore, feed temperature mainly influences the amount of heat withdrawn from the bottom of the tower. Its influence on the nonlinearity of the heat transfer process is not very significant. Feed composition does influence model nonlinearity since it will affect the fluid enthalpy and mass and energy transfer coefficients. But the influence will not be significant.

The most important factor influencing the nonlinearities of the tower is the feed flow-rate. The variation of feed flow-rate will lead to changes of mass and energy hold-up in every tray, and result in significantly changing process dynamics. As an example Fig.2 shows two rather different pumparound step responses for two operating points with different feed flow-rate. Therefore, feed flow-rate should be selected to characterize the nonlinearities for the distillation column in question.

In industrial units a very important factor influencing the tower dynamics is coke accumulation and equipment wearing in the tower. This case can only be studied based on the data from industry unit and will not be discussed in this paper.

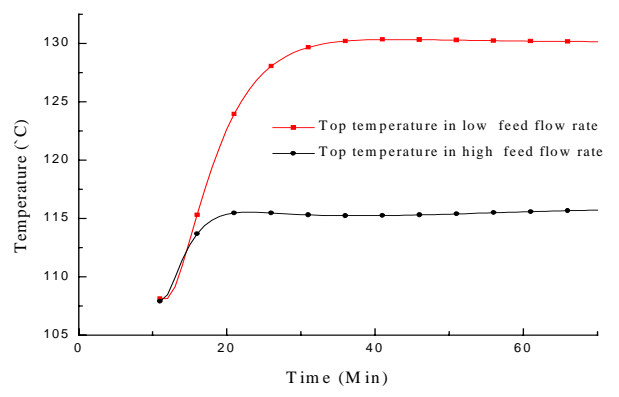

Fig. 2. Top pump-around flow-rate increased by $15 \%$. High feed flow-rate means a $50 \%$ increase compared to the low feed flow-rate.

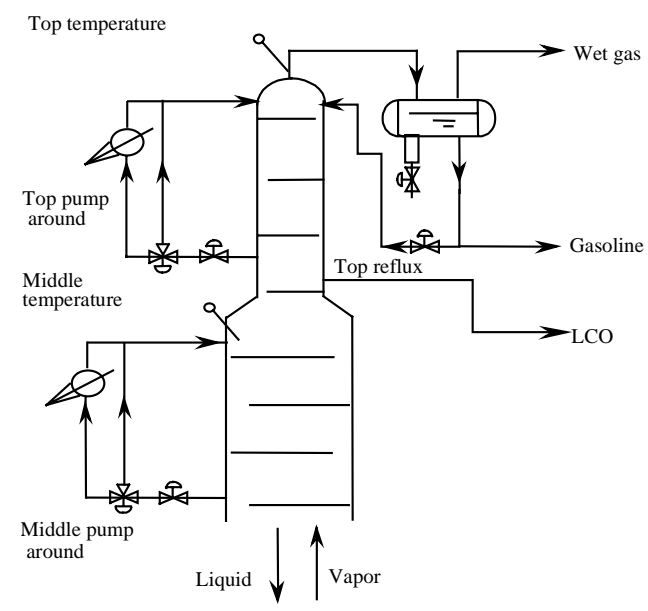

Fig. 3. Top part of FCCU fractionator

\subsection{FCCU Fractionator Control Structure}

A FCCU fractionator is an essential unit for separating gasoline and diesel from feedstock from an upstream riser reactor. Its main products, gasoline and LCO (light cycle oil), are distillated from the top of the tower as shown in Fig. 3. The product qualities of these two products are the main variables we are concerned with. Top and middle temperatures are assigned to be the two controlled variables. The process variables directly influencing the controlled variables include the "T" valves in the top and middle pump-around system, reflux flow-rate, top pump-around flow-rate and middle pump-around flow-rate. In this investigation, these five variables are used as the manipulated variables to control two product qualities represented here by top and middle temperatures.

For the other process variables, such as bottom level and temperature, we use single-input, singleoutput PID controller.

\subsection{Local Model Structure}

The top part of the FCCU fractionator may be divided into two disjunct compartments wherefrom we produce gasoline and LCO, respectively. Thus, corresponding to (7) and (8), we chose a local model as follows.

$$
\begin{aligned}
& \dot{x}=A x+B u+F v \\
& y=C x
\end{aligned}
$$

The state variable $x=\left(x_{1}, x_{2}, x_{3}, x_{4}\right)^{\mathrm{T}}$, output variable $y \in\left(x_{1}, x_{2}\right)^{\mathrm{T}}$, manipulated variable $u=\left(u_{1}, u_{2}, u_{3}, u_{4}\right.$, $\left.u_{5}\right)^{\mathrm{T}}$ and measurable disturbance $v$ are defined in the nomenclature list. Supposing the characteristic variable is $v$, the global model corresponding to (4) is formulated as 


$$
\begin{aligned}
& \dot{x}=\sum_{i=1}^{N} A_{i} w_{i}(v) x+\sum_{i=1}^{N} B_{i} w_{i}(v) u+\sum_{i=1}^{N}+F_{i} w_{i}(v) v \\
& y=\sum_{i=1}^{N} C_{i} x w_{i}(v)
\end{aligned}
$$

$x, u$ and $y$ are variables around steady state values. $w_{i}(v)$ is given by

$$
\begin{gathered}
w_{i}(v)=\frac{\rho_{i}(v)}{\sum_{i=1}^{d} \rho_{i}(v)} \\
\rho_{i}(v)=\exp \left(-\frac{1}{2}\left(\frac{v-\overline{v_{i}}}{\gamma_{i} \Delta v_{i}}\right)^{2}\right) \\
\bar{v}_{i}=0.5\left(v_{i, \min }+v_{i, \max }\right), \Delta v_{i}=0.5\left(v_{i, \max }-v_{i, \text { min }}\right)
\end{gathered}
$$

and the $\mathrm{i}$-th regime is characterized by the intervals $\left[v_{i, \min }, v_{i, \max }\right]$. In addition, the parameter $\gamma_{i}$ determines the smoothness.

The range of feed flow-rate is divided into 3 operating regimes as shown in Fig.4. The variation span of the feed flow-rate in each regime will be $17 \%$ of its normal value i.e. $200 \mathrm{kmol} / \mathrm{h}$.

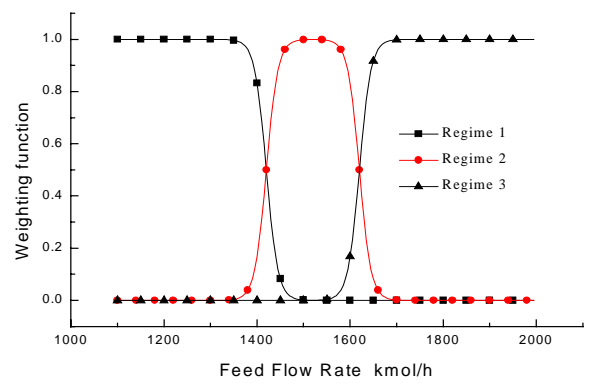

Fig. 4. Interpolation function for the three regimes spanned by the feed flow-rate

\subsection{Parameter Estimation}

Data used for local model parameter estimation is obtained from a rigorous fractionator dynamic model that has been fitted to real plant data (Cong et al. 1998). A pseudo-random binary sequence is used as the excitation signal, and the resulted data is passed through a low-pass filter with a time constant of 5 minutes, By this, we ensure that the data gives a good basis for identifying a control-relevant model(Shook et. al., 1992). In this case a controlrelevant model means a model with good prediction quality on the time horizon of the MPC, i.e. 0-10 minutes. Model validation results are shown in Fig. 5 , where 5 minutes predictions are compared to data from the rigorous model.

For this exposition we have chosen to compare a local model based on the linear model valid in regime 3 to the multi model. The results are quite as expected. The models have similar prediction capability in regime 3 whereas the multi-model is clearly the better in regime 1 and 2 .

\section{NONLINEAR MPC CONTROL}

In industrial applications, most control system will have more inputs than outputs. This brings to us a problem of how to optimize the excessive manipulated variables. We solve this by including ideal resting values (IRV) in the objective. Our MMPC problem can be described as follows.

$$
\begin{aligned}
& \underset{U}{\operatorname{Min}} J=\left\{\frac{1}{2} \sum_{i=p_{1}}^{p_{2}} \|\left[\hat{y}(k+i)-y_{s p}(k+i)\right]\right\rangle \|_{W}^{2}+ \\
& \left.\frac{1}{2} \sum_{j=0}^{M}\left\|u(k+j)-u_{I R V}\right\|_{Q^{\prime}}^{2}\right\}+\frac{1}{2} \sum_{j=0}^{M}\|u(k+j+1)-u(k+j)\|_{Q_{1}}^{2} \\
& x(k+1)=\sum_{i=1}^{N} G_{i} w_{i}(v) x(k)+\sum_{i=1}^{N} H_{i} w_{i}(v) u(k) \\
& +\sum_{i=1}^{N} R_{i} w_{i}(v) v(k) \\
& x(k)=\hat{x}(k) \\
& y_{\text {min }} \leq y(k+i) \leq y_{\max }, \quad i=p_{1}, p_{1}+1, \ldots, p_{2} \\
& u_{\min } \leq u(k+j) \leq u_{\max }, \quad j=0,1,2, \ldots, M \\
& u(k+j)=u(k+M), \quad M \leq j \leq p_{2} \\
& |\Delta u(k+j)| \leq u_{d}
\end{aligned}
$$
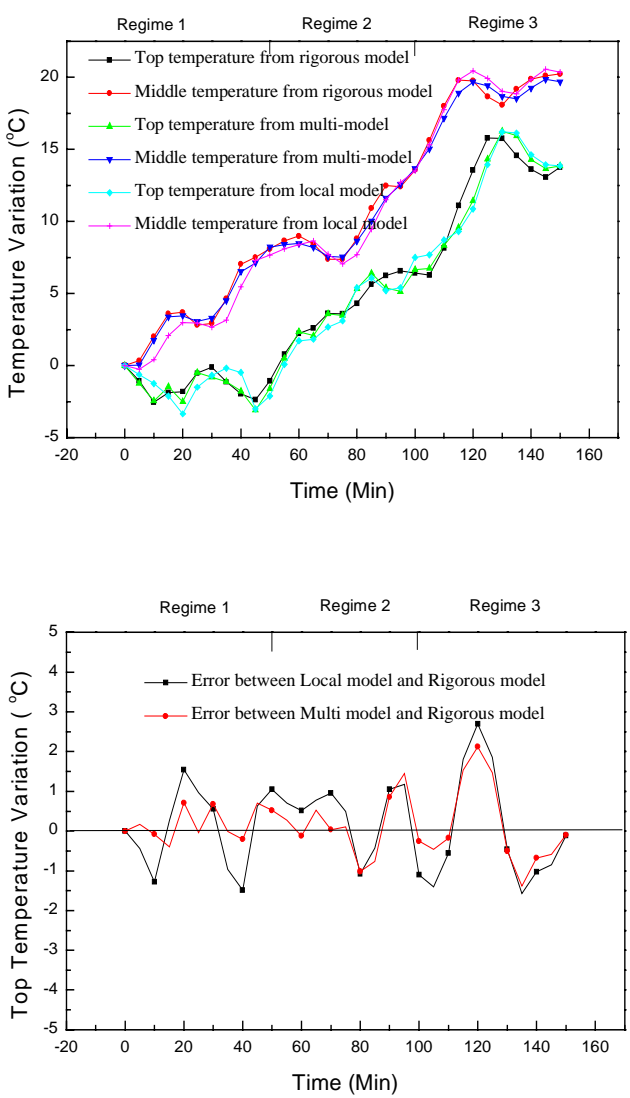

Fig. 5. 5 Minutes predictions when 5 inputs vary as a pseudo random binary signal and feed flowrate varies from 1320 to $1720 \mathrm{kmol} / \mathrm{h}$.

$y_{\mathrm{sp}}$ is the setpoint of controlled variable, $y_{\mathrm{r}}$ is the measured output, $y_{\min }, y_{\max }$ are boundary constraints on $y, u_{\min }$ and $u_{\max }$ are the boundary constraints on $u, u_{\mathrm{d}}$ is the velocity constraint on $u, p_{1}, p_{2}$ are 
predictive horizons and $M$ is the control horizon. The equality constraint formulations are based on the discrete form of (9) and (10). The sampling time is 30 seconds. $\hat{x}$ is the estimated state from the following state estimator.

$$
\begin{aligned}
& \hat{x}(k+1)=\sum_{j=1}^{N} G_{j} w_{j}(v) \hat{x}(k)+\sum_{i=1}^{N} w_{i}(v) K\left(y_{r}(k+1)-\right. \\
& \left.C_{i} \sum_{j=1}^{N} G_{j} w_{j}(v) \hat{x}(k)\right)+\sum_{i=1}^{N} H_{i} w_{i}(v) u(k)+\sum_{i=1}^{N} R_{i} w_{i}(v) v(k)
\end{aligned}
$$

$K$ is chosen such that the eigenvalue of the state estimator is approximately 5 time steps. The nonlinear programming problem is solved through a feasible sequential quadratic programming (FSQP) algorithm (Lawrence et. al., 1997).

\section{$4 \quad$ RESULTS}

The MMPC is evaluated on a set of realistic simulation scenarios. They include temperature setpoint change (tracking process), feed flow-rate, feed temperature variation and feed composition (disturbance resistance). The control problems are simulated with values $p_{1}=1, p_{2}=20, M=2$. Boundary limit $u_{\max }=100, u_{\min }=0$, and $u_{\mathrm{d}}=1$.

Eig. 6 compares MMPC and Single (local) Model Based Predictive Controller (SMPC), i.e. linear MPC after a setpoint change. SMPC works very well within the local regime where the local model is valid. It does, however, not give good control performance when operation moves away from this regime. MMPC gives good control performance in all the operating regimes.

Eig. 7 shows performance to measurable disturbance changes i.e. feed flow-rate changes. Fig. 8 and Fig. 9 show performance to unmeasurable disturbances. For all these disturbances, MMPC improves performances vs. SMPC.

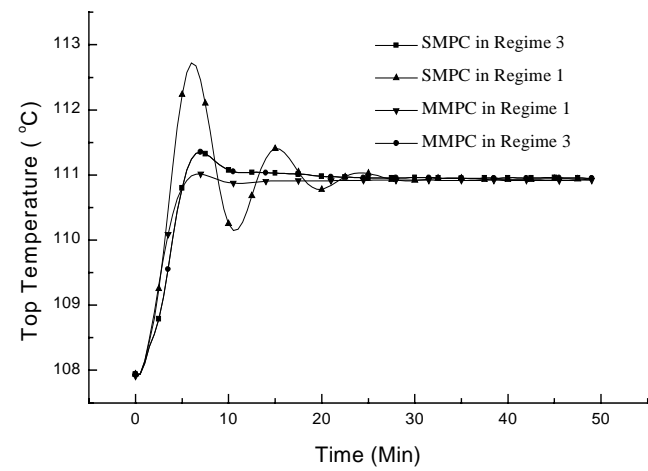

Fig. 6. Top temperature setpoint increased by $3{ }^{\circ} \mathrm{C}$ and the local model used by SMPC is based on regime 3 .

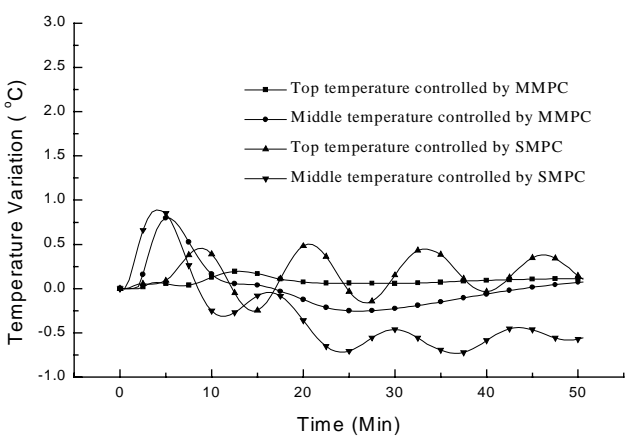

Fig. 7. Feed flow-rate increased by $50 \mathrm{kmol} / \mathrm{h}$ as a measurable disturbance.

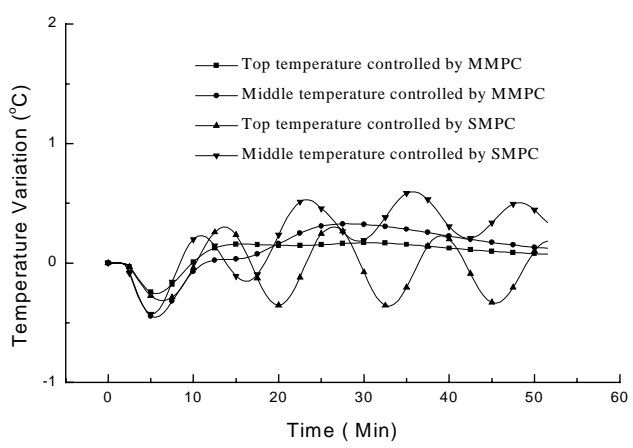

Fig. 8. Feed temperature decreased by $20^{\circ} \mathrm{C}$ as an unmeasurable disturbance.

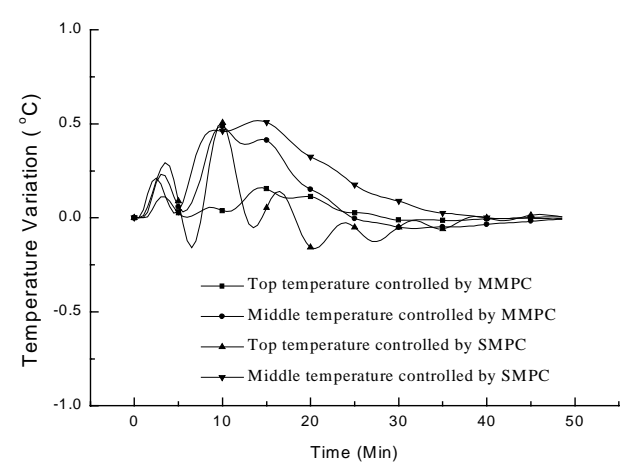

Fig. 9. Feed composition became heavier as an unmeasurable disturbance

The MMPC's coordinated control ability is shown in Fig. 10, moving both controlled variables and sorhe of the manipulated variables to their setpoint if it is possible. When there is more inputs than outputs, MMPC will first make full use of all available control efforts to drive the controlled variables to their setpoint regardless where the IRVs of manipulated variables are located. After the controlled variables arrive at their setpoint, MMPC coordinates some of the manipulated variables to their IRV slowly. The manipulated variables to be coordinated are selected depending on their economic importance. 


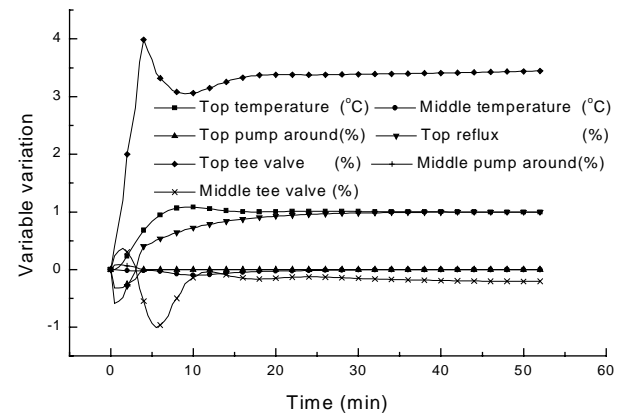

Fig. 10. The setpoint top temperature increased by $1{ }^{\circ} \mathrm{C}$, The ideal resting value of top reflux flow-rate increased by $1 \%$.

\section{DISCUSSION AND CONCLUSION}

MMPC is introduced as an option to migrate from linear to nonlinear MPC in a graceful fashion. By using linear local models the well-proven properties of linear MPC are retained as far as possible. If, for instance, the system operate within one regime the MMPC will in actual fact operate like a linear MPC. It is only during transients that the nonlinear capabilities of the MMPC will be activated. Further, refining MMPC is conceptually straightforward since new local models may be added in operating regimes where the existing multi-model is deficient. In the simulating example we directly manipulate the control inputs. We world have manipulated the setpoints of basic control loops instead. This would most probably reduce the necessity for nonlinear MPC.

This investigation has indicated that multi-model based MPC can be an attractive way to introduce nonlinear MPC in a graceful fashion.

\section{NOMENCLATURE:}

$C_{\mathrm{p}}$ specific heat, $\mathrm{kJ} /\left(\mathrm{kmol} .{ }^{\circ} \mathrm{C}\right)$

$H$ liquid enthalpy, $\mathrm{kJ} / \mathrm{kmol}$

$h$ vapor enthalpy, $\mathrm{kJ} / \mathrm{kmol}$

$K_{\text {st }}$ heat transfer coefficient, $\mathrm{kJ} /{ }^{\circ} \mathrm{C} . \mathrm{h} . \mathrm{m}^{2}$

$L$ liquid flow-rate, $\mathrm{kmol} / \mathrm{h}$

$M$ mass holdup, kmol

$Q$ heat loss, $\mathrm{kJ} / \mathrm{h}$

$T$ temperature, ${ }^{\circ} \mathrm{C}$

$T_{\mathrm{si}}$ input temperature of heat exchange medium, ${ }^{\circ} \mathrm{C}$

$T_{\text {so }}$ output temperature of heat exchange medium, ${ }^{\circ} \mathrm{C}$

$u$ manipulated variable, valve position, fraction

$u_{1}$ top pump-around flow-rate, $\mathrm{kmol} / \mathrm{h}$

$u_{2}$ top reflux, $\mathrm{kmol} / \mathrm{h}$

$u_{3}$ top tee valve position, fraction

$u_{4}$ middle pump-around flow-rate, $\mathrm{kmol} / \mathrm{h}$

$u_{5}$ middle tee valve position, fraction

$v$ measurable disturbance, vapor phase flow-rate, $\mathrm{kmol} / \mathrm{h}$.

$x_{1}$ top vapor temperature, ${ }^{\circ} \mathrm{C}$

$x_{2}$ middle vapor temperature, ${ }^{\circ} \mathrm{C}$

$x_{3}$ top exchanger outlet temperature, ${ }^{\circ} \mathrm{C}$ $x_{4}$ middle exchanger outlet temperature, ${ }^{\circ} \mathrm{C}$

$x$ state variable

$y$ controlled variable

Greek letters

$\Omega$ heat transfer area, $\mathrm{m}^{2}$

$\rho$ density, $\mathrm{kg} / \mathrm{m}^{3}$

\section{Subscripts \\ $i \quad$ component $i$ \\ $j$ tray No. \\ $p$ pump-around}

Superscripts

$L$ liquid phase

$V$ vapor phase

\section{REFERENCE}

Benallou, A., D. E. Seborg and D. A. Mellichamp (1986). Dynamic compartment model for separation process. A.I.Ch.E. J. 32, pp.10671078.

Cong, S.-B., Yuan Pu and Shen Fu (1998). An integrated non-equilibrium dynamic model of petroleum distillation column. Submitted to Chemical Engineering Science.

Cong, S.-B., Yuan Pu and Shen Fu (1996). Dynamic model simplification of heat exchanger. Petroleum Processing and Petrochemicals 27(10), pp.5-9.

Johansen, T. A. and B. A. Foss (1997). Operation regime based process modeling and identification. Computers Chem. Engng. 21(2), pp.159-176.

Lawrence, C., J. L. Zhou and A. L. Tits (1997) User's guide for CFSQP version 2.5. Institute for System Engineering, University of Maryland, College Park, MD 20742.

Murray-Smith, R. and T. A. Johansen (1997). Multiple model approaches to modeling and control. Taylor \&Francis, London.

Qin, S. J. and T. A. Badgwell (1996). An overview of industrial model predictive control technology. $C P C V$, Lake Tahoe, USA.

Qin, S. J. and T. A. Badgwell (1998). An overview of nonlinear model predictive control application. Ascona.

Shook, D. S., C. Mohtadi and S. L. Shah (1992). A control relevant identification strategy for GPC. IEEE Transactions on Automatic Control, 37 (7), pp. 975-980 